\title{
COMPARATIVE SCANNING ELECTRON MICROSCOPIC STUDY OF THE EFFECT OF DIFFERENT DENTAL CONDITIONERS ON DENTIN MICROMORPHOLOGY
}

\author{
Alexandre Henrique SUSIN ${ }^{1}$, Luana Severo ALVES ${ }^{2}$, Gliciana Piovesan de MELO ${ }^{2}$, Tathiane Larissa LENZI ${ }^{2}$
}

1- DDS, MSc, PhD, Professor, Department of Restorative Dentistry, Federal University of Santa Maria, Santa Maria, RS, Brazil; Professor. Postgraduate Program in Restorative Dentistry, Dental School of Araraquara, State University of Sao Paulo, Araraquara, SP, Brazil.

2- DDS, Graduate in Dentistry, Federal University of Santa Maria, Santa Maria, RS, Brazil.

Corresponding address: Prof.Dr. Alexandre Henrique Susin - Departamento de Odontologia Restauradora - UFSM - Rua Mal. Floriano, 1184 - primeiro andar - 97015-372 - Santa Maria, RS, Brazil. Phone: 5555 3220-9281 or 5555 3226-5121 (mob. 55 55 8407-9729).

e-mail: ahsusin@brturbo.com.br

Received: February 12, 2007 - Modification: August 20, 2007 - Accepted: December 05, 2007

\begin{abstract}
7

his study evaluated comparatively by scanning electron microscopy (SEM) the effect of different dental conditioners on dentin micromorphology, when used according to the same protocol. Forty dentin sticks were obtained from 20 caries-free third human molars and were assigned to 4 groups corresponding to 3 conditioners (phosphoric acid 37\%, Clearfil SE Bond and iBond) and an untreated control group. After application of the conditioners, the specimens were immersed in $50 \%$ ethanol solution during $10 \mathrm{~s}$, chemically fixed and dehydrated to prepare them to SEM analysis. In the control group, dentin surface was completely covered by smear layer and all dentinal tubules were occluded. In the phosphoric acid-etched group, dentin surface was completely clean and presented exposed dentinal tubule openings; this was the only group in which the tubules exhibited the funnel-shaped aspect. In the groups conditioned with Clearfil SE Bond primer and iBond, which are less acidic than phosphoric acid, tubule openings were occluded or partially occluded, though smear layer removal was observed. SE Bond was more efficient in removing the smear layer than iBond. In the Clearfil SE Bond group, the cuff-like aspect of peritubular dentin was more evident. It may be concluded all tested conditioners were able to change dentin morphology. However, it cannot be stated that the agent aggressiveness was the only cause of the micromorphological alterations because a single morphological pattern was not established for each group, but rather an association of different aspects, according to the aggressiveness of the tested conditioner.
\end{abstract}

Key words: Adhesive system. Self-etching adhesives. Dental conditioners.

\section{INTRODUCTION}

Adhesive dentistry has undergone remarkable changes since the introduction of phosphoric acid as an enamel conditioner ${ }^{1}$ and the same principles still guide adhesion, that is, partial demineralization of the dental structure and impregnation of the etched area with resin monomers. The technique of dentin hybridization and the concept of hybrid layer ${ }^{8}$ caused a significant advance in adhesive dentistry. From the 90's until the current days, the adhesive technology incorporating new components had provided a great progress and unquestionable clinical and laboratorial improvements in cavity sealing, reduction of microleakage and increase of tensile bond strength ${ }^{11,14}$.

The adhesive systems can be classified in two major groups, according to the need or not of acid etching prior to the impregnation of the dental substrate with resin monomers. The materials that require previous acid etching are classified as total-etch adhesive systems, whereas those that perform the conditioning of the dental surface simultaneously to resin monomer interdiffusion are classified as self-etching adhesive systems $\mathrm{s}^{4,12,15}$.

The total-etch adhesive systems use a strong acid agent, like phosphoric acid, that removes the smear layer and demineralizes enamel and dentin, exposing the collagen fibrils and increasing the surface free energy. The presence of hydrophilic monomers, which are agents that promote adhesion in these materials, makes their use critical regarding the moisture that remains in the dental substrate after cavity rinsing and drying. Overdrying causes tissue dehydration, especially in dentin, and can compromise the performance of these adhesive systems $s^{6,10,16,17}$.

The self-etching adhesive systems have a significantly lower $\mathrm{pH}$ than that of total-etch adhesive systems because the acidic monomer present in their composition propitiates changes in the substrate, smear layer incorporation/ 
dissolution and surface demineralization simultaneously to resin monomer impregnation. The use these materials eliminates the need of phosphoric acid etching, water rinsing and air drying as separate steps of the bonding protocol. It is assumed that self-etching adhesive systems form a zone of interdiffusion free of non-hybridized areas because the entire depth of dentin demineralization is simultaneously filled with resin monomers. Same efficacy cannot be warranted when conventional etch-and-rinse systems are used $^{12,15}$.

The study of hybrid layer morphology and the effect of conditioners on dentin is an important resource to analyze the characteristics of dental substrate and to understand which factors can contribute to improve the effectiveness of adhesive systems. The purpose of this study was to evaluate comparatively by scanning electron microscopy (SEM) the effect of different conditioners on dentin micromorphology, when used according to the same protocol.

\section{MATERIALAND METHODS}

Twenty caries-free third molars extracted from young patients for therapeutic indication were used in this study. The teeth were sectioned with a diamond saw (ISOMET 1000; Buheler, Lake Bluff, IL, USA) 3 mm below the occlusal third to remove the cusps. Two-millimeter-thick dentin disks were obtained by sectioning the crowns parallel to the occlusal surface. Deep dentin was chosen because this region favors adhesive diffusion through the demineralized dentin and hence provides the best conditions for resin monomer infiltration, as the number and diameter of tubules in coronal dentin increases significantly from the middle to the deepest layer near the pulp ${ }^{11}$. The disks were polished with 600-grit silicon carbide paper for $30 \mathrm{~s}$ to produce a standardized smear layer similar to that formed on burprepared dentin.

The disks were bisected in a mesiodistal direction to obtain two sticks from each disk. The tested conditioners are shown in figure 1 . The dentin sticks were randomly assigned to 4 groups $(n=10)$, according to the treatment protocol: no treatment (control), 37\% phosphoric acid gel, iBond and Clearfil SE Bond conditioners. Prior to application of the conditioners, the dentin sticks were marked on the laterals sides and on the opposite side with a diamond saw in order to facilitate their fracturing for SEM analysis. The specimens were submitted to the treatment with the conditioners as displayed in Table 1.

Immediately after conditioner application and rinsing, the specimens were immersed in $2.5 \%$ glutaraldehyde in 0.1 $\mathrm{M}$ sodium cacodylate buffer for $6 \mathrm{~h}$ for fixation, thereafter rinsed with $0.1 \mathrm{M}$ sodium cacodylate and then rinsed for 1 min with distilled water. Then, the dentin sticks were dehydrated in ethanol ascending degrees as follows: $50 \%$, $70 \%$ and $90 \%$ for $5 \mathrm{~min}$ each and then $100 \%$ for $3 \mathrm{~h}$. Next, the specimens were ultrasonicated for $15 \mathrm{~min}$ in $100 \%$ ethanol and then carefully fractured manually on the middle and bonded in metallic platforms with cyanoacrylate. Half of the specimens were positioned in such a way that they could

FIGURE 1- Conditioners, manufacturers and respective chemical compositions

\begin{tabular}{|cccc|}
\hline Conditioners & Manufacturer & Composition & Batch \# \\
\hline $\begin{array}{c}\text { 37\% phosphoric acid gel } \\
\text { 3M/ESPE, St.Paul, MN, }\end{array}$ & $37 \%$ phosphoric acid gel & $3 \mathrm{BC}$ \\
$\begin{array}{c}\text { USA } \\
\text { iBond all-in-one self-etching }\end{array}$ & Heraeus Kulzer, & UDMA, 4 Met, acetone, water, & 020044 \\
$\begin{array}{l}\text { Clearfil SE Bond self-etching } \\
\text { adhesive system with acidic } \\
\text { primer }\end{array}$ & Kuraray Co., Osaka, & glutaraldehyde, camphorquinone. & MDP, HEMA, hydrophilic \\
& Japan & dimethacrylate, photoinitiator, water. & \\
\hline
\end{tabular}

TABLE 1- Groups, conditioners and respective treatment protocols

Group $(n=10) \quad$ Conditioners $\quad$ Treatment Protocol

\begin{tabular}{|c|c|c|}
\hline & None & The specimens received no treatment. \\
\hline & $37 \%$ phosphoric acid & $\begin{array}{l}\text { Application for } 20 \mathrm{~s} \text {, immersion in } 50 \% \text { ethanol } \\
\text { aqueous solution for } 5 \mathrm{~s}\end{array}$ \\
\hline & iBond & $\begin{array}{l}\text { Air/water spray rinsing for } 30 \mathrm{~s} \text {, drying of the etched } \\
\text { surface with absorbent paper. }\end{array}$ \\
\hline
\end{tabular}

$4 \quad$ Clearfil SE Bond 
be observed in a cross-sectional view, while the others were positioned to permit a longitudinal view.

The dentin specimens were sputter-coated with gold (SCD 030; Blazers AG, Balzers, Liechtenstein) and observed under a scanning electron microscope (Philips XL Series XL 20; Philips, Eindhoven, The Netherlands) at $25 \mathrm{kV}$. The images were captured at magnifications of x8,000 and x32,000.

The overall aspect of etched dentin surface was evaluated with regards to depth of intertubular demineralization (as measured by Image Tool Software 2.00 (UTHSCSA, San Antonio, TX, USA), cuff-like aspect of peritubular dentin, funnel-shaped aspect of dentinal tubules, presence/absence of smear layer, presence/absence of smear plugs (percentage of occluded, partially occluded and open tubules), in order to compare the conditioners and their $\mathrm{pH}$ values.

ANOVA was applied to compare the means of depth of demineralization and Tukey's test $(p=0.05)$ was applied to determine the significance of detected differences among the groups.

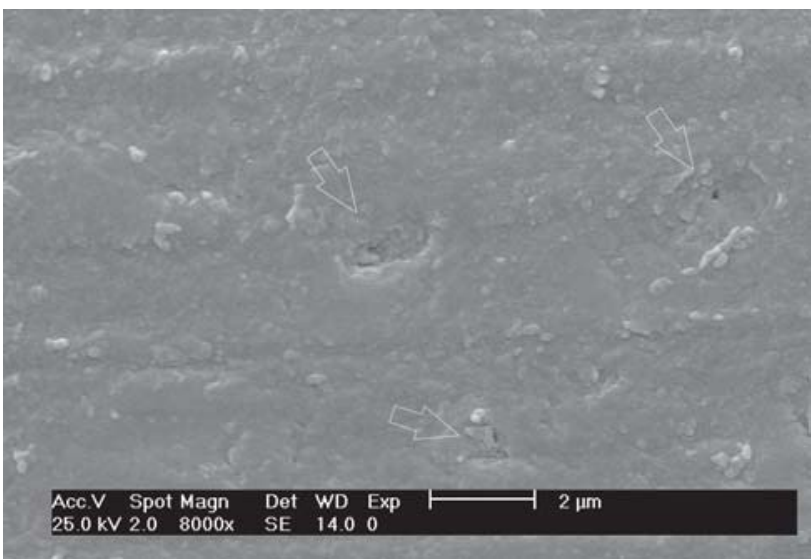

FIGURE 2- SEM micrograph of group 1 (no treatment) Occlusal view of dentinal tubules. Observe the dentin surface completely covered by debris (smear layer) and occluded tubule openings (arrows) $(x 8,000)$

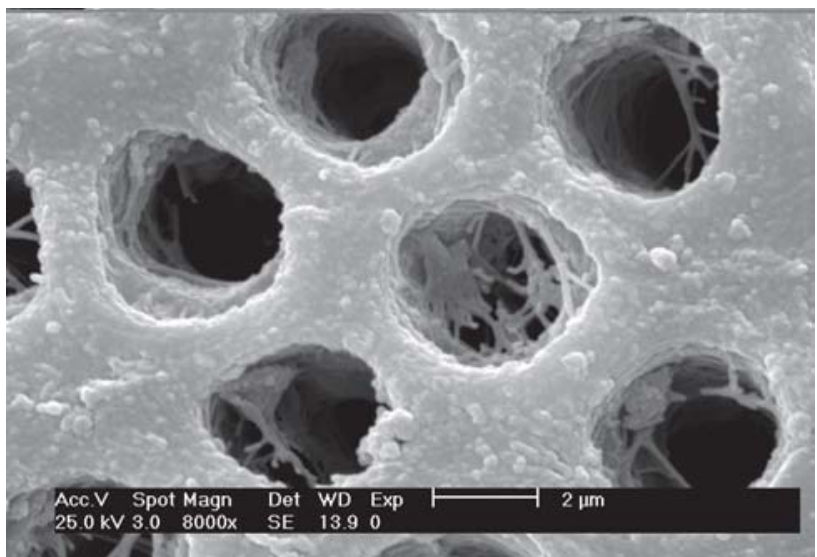

FIGURE 4- SEM micrograph of group 2 (37\% phosphoric acid etching) - Occlusal view of one dentinal tubule. Observe the dentin surface free of smear layer and ope tubule $(x 8,000)$

\section{RESULTS}

The analysis of cross-sectional and longitudinal specimens under SEM showed that the tested conditioners promoted alterations in dentin micromorphology. The results demonstrated that different conditioners can promote similar effects on surface micromorphology.

Figures 2 to 9 show dentin micromorphology after application of each conditioner. Specimens that received no treatment and those etched with phosphoric acid (groups 1 and 2, respectively) will be first compared in order to characterize phosphoric acid aggressiveness on dentin, which will be used as parameter for comparison to the other groups.

All conditioners used in this study were effective in removing the smear layer completely, but not all of them were able to remove the smear plugs. The results of group 1 (no treatment) showed the expected pattern of dentin substrate after polishing. The smear layer and smear plugs were maintained over dentin occluding $100 \%$ of the tubules. In group 2 (phosphoric acid etching), a depth of

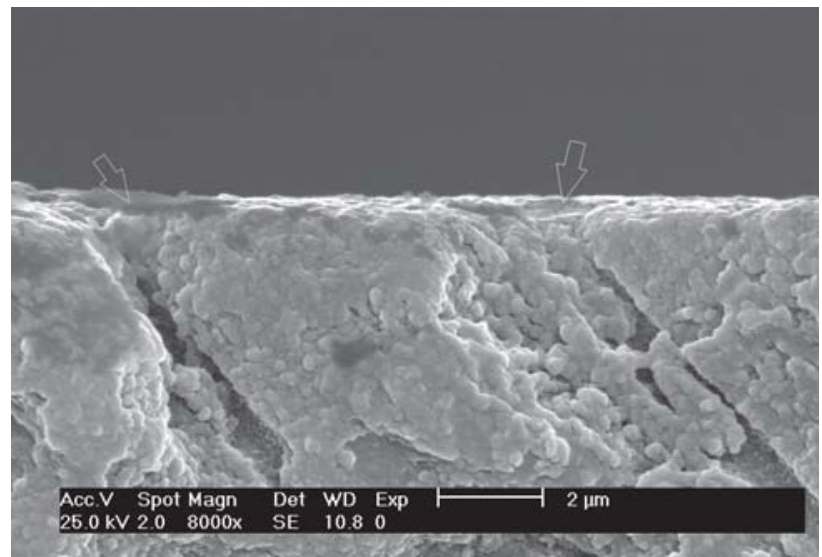

FIGURE 3 - SEM micrograph of group 1 (no treatment) Lateral view of the dentinal tubules. Observe the tubules filled in with debris (smear plugs) (arrows) produced by 600 -grit silicon paper grinding $(x 8,000)$

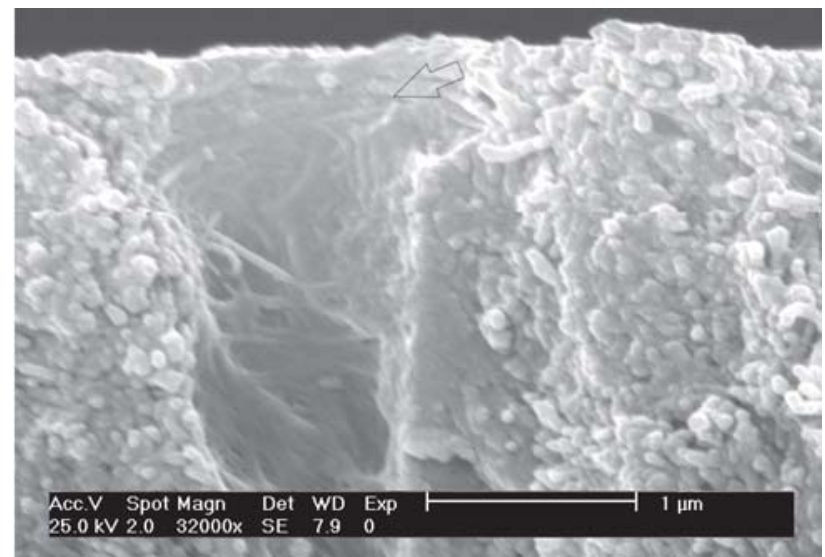

FIGURE 5- SEM micrograph of group 2 (37\% phosphoric acid etching) - Lateral view of dentin surface. Observe the tubule openning free of debris (arrow) and with a funneled aspect. $(\mathrm{x} 32,000)$ 


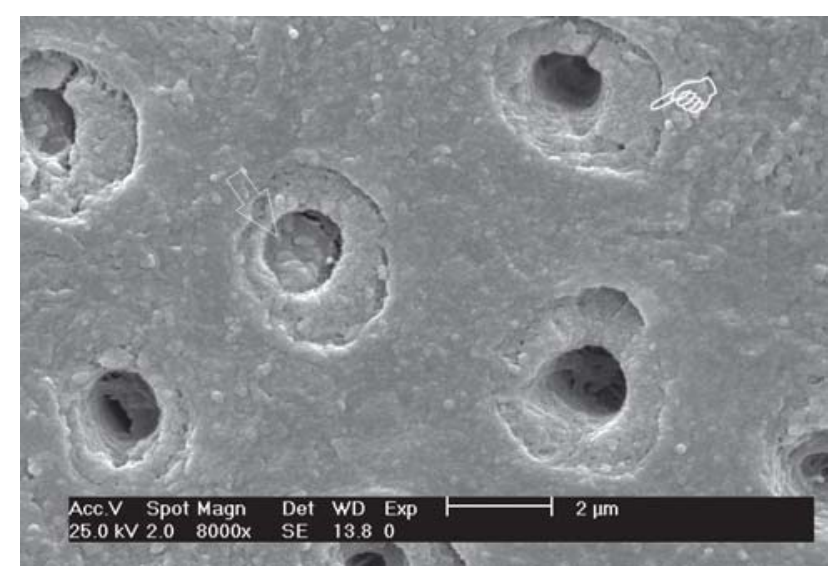

FIGURE 6 - SEM micrograph of group 3 (iBond) - Occlusal view of dentin surface. Observe the cuff-like aspect of the peritubular dentin (arrowhead) and some partially occluded tubules (arrow) $(x 8,000)$

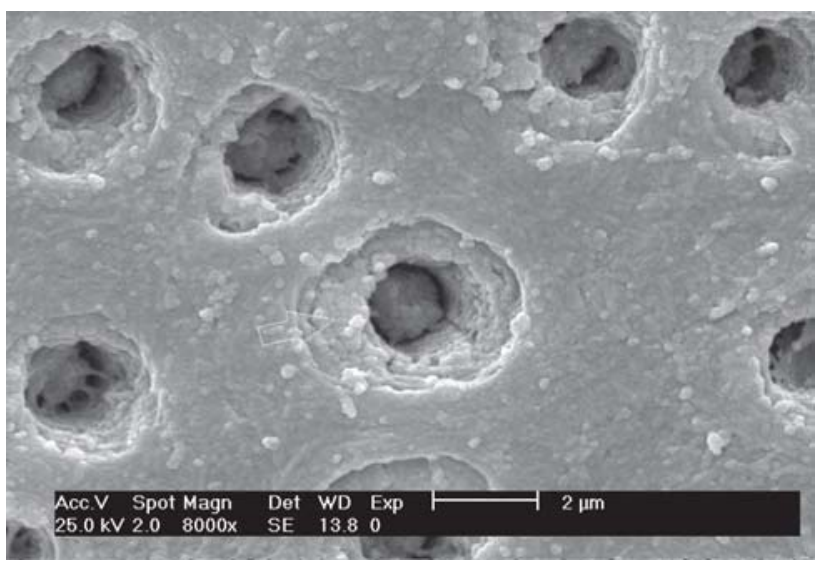

FIGURE 8- SEM micrograph of group 4 (Clearfil SE Bond) Occlusal view of dentin surface. Observe the cuff-like aspect of the peritubular dentin (arrow) and the aperture of the exposed dentinal tubules $(x 8,000)$

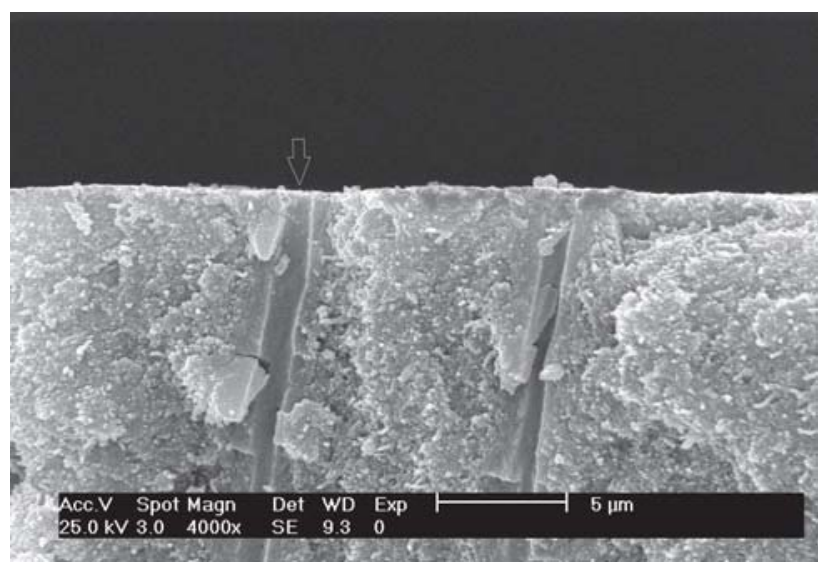

FIGURE 7- SEM micrograph of group 3 (iBond) - Lateral view of dentin tubules. Observe a totally opened (arrow) and a occluded dentinal tubule $(x 8,000)$

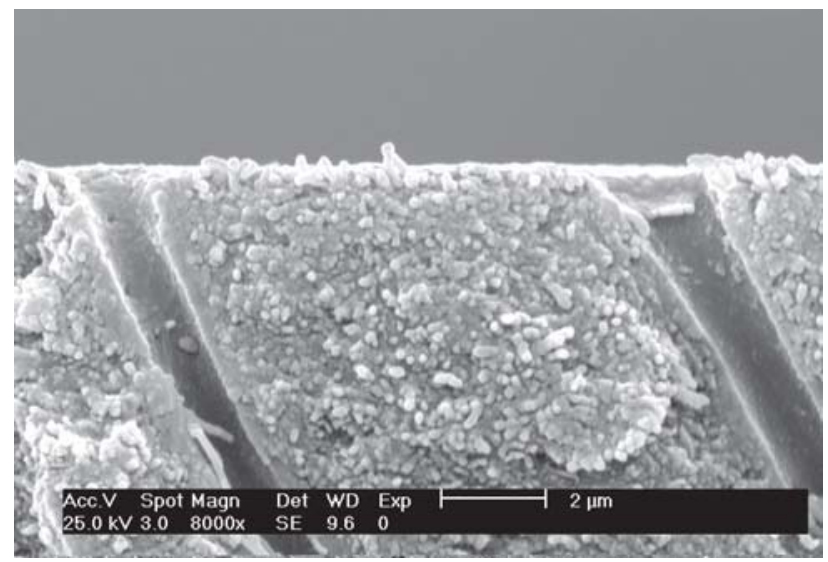

FIGURE 9- SEM micrograph of group 4 (Clearfil SE Bond) Lateral view of the dentinal tubules. Observe the dentin surface free of smear layer and opened dentinal tubules $(x 8,000)$

TABLE 2- Results according to the evaluated criteria

\begin{tabular}{|c|c|c|c|c|c|c|c|c|c|c|}
\hline \multirow[t]{2}{*}{ Group } & \multirow[t]{2}{*}{ Conditioner } & \multirow[t]{2}{*}{$\mathrm{pH}$} & \multirow[t]{2}{*}{$\begin{array}{c}\text { Depth of } \\
\text { intertubular } \\
\text { demineralization }\end{array}$} & \multirow[t]{2}{*}{$\begin{array}{c}\text { Tukey } \\
\text { grouping }\end{array}$} & \multirow[t]{2}{*}{$\begin{array}{l}\text { Cuff-like } \\
\text { aspect of } \\
\text { peritubular } \\
\text { dentin }\end{array}$} & \multirow[t]{2}{*}{$\begin{array}{l}\text { Funnel- } \\
\text { shaped } \\
\text { tubules }\end{array}$} & \multirow[t]{2}{*}{$\begin{array}{l}\text { Smear } \\
\text { layer }\end{array}$} & \multicolumn{3}{|c|}{$\begin{array}{c}\text { Smear Plug } \\
\% \text { of occluded (O), } \\
\text { partially occluded } \\
\text { (PO) and open (OP) } \\
\text { tubules }\end{array}$} \\
\hline & & & & & & & & $\mathrm{O}$ & $\mathrm{PO}$ & $\mathrm{OP}$ \\
\hline 1 & None & - & Absent (score c) & - & No & No & Yes & 100 & - & - \\
\hline 2 & $\begin{array}{l}\text { Phosphoric } \\
\text { acid }\end{array}$ & 0.5 & $\begin{array}{c}1.83 \mu \mathrm{m}( \pm 0.4) \\
(\text { score a) }\end{array}$ & * & No & Yes & No & - & - & 100 \\
\hline 3 & i Bond & 1.9 & $\begin{array}{c}<0.5 \mu \mathrm{m} \text { or absent } \\
\text { (score c) }\end{array}$ & $* * *$ & Yes & No & No & 33.3 & 27.2 & 39.5 \\
\hline 4 & CSEB (primer) & 1.9 & $\begin{array}{c}0.8 \mu \mathrm{m}( \pm 0.4) \\
(\text { score b) }\end{array}$ & $* *$ & Yes & No & No & 24.3 & 25.2 & 50.5 \\
\hline
\end{tabular}

DMS tukey's test $(p=0.05)=0.33$. Scores: $a=>1.51 \mu \mathrm{m}$ of depth of demineralization; $b=0.5$ to $1.0 \mu \mathrm{m}$ of depth of demineralization; and $c=<0.5 \mu \mathrm{m}$ or absence of depth of demineralization. 
demineralization of $1.83 \mu \mathrm{m}$ was observed and the dentinal tubules had a funneled aspect and $100 \%$ of occluded openings. In group 3 (iBond), dentin demineralization was not observed. The funneled aspect was absent and the cufflike peritubular dentin aspect could be observed in the open dentin tubules. In group 4 (Clearfil SE Bond primer), a depth of demineralization of $0.80 \mu \mathrm{m}$ was observed and approximately $50 \%$ of the dentin tubules were open and peritubular dentin exhibited a cuff-like aspect.

The results of each group according to the evaluated criteria are shown in Table 2.

\section{DISCUSSION}

The use of self-etching adhesive systems has increased remarkably in clinical practice ${ }^{13}$ due to technique simplification and reduction of the number of clinical steps. In addition, total-etch adhesive systems may produce imperfections in the hybrid layer due to dentin dehydration because acid etching and drying of the cavity are critical points of the bonding protocol. Self-etching adhesive systems contain acidic monomers that allow them promoting adhesion to smear-covered dentin. The acidic monomers are able to infiltrate and incorporate the smear layer within the hybrid layer ${ }^{5,13}$.

One of the greatest concerns about these materials refers to their $\mathrm{pH}$. It is discussed whether the $\mathrm{pH}$ of self-etching adhesive systems is acid enough to produce adequate demineralization compared to etch-and-rinse adhesive systems. The $\mathrm{pH}$ is a key factor to determine the penetration potential throughout dentin and the depth of demineralization, and it is clinically relevant for the postoperative sensitivity in deep cavities and for prevention of the water tree process ${ }^{2,5,9,11,12}$.

During their evolution, adhesive systems have undergone significant modifications in their chemical composition, such as the addition of hydrophilic monomers, acidic monomers and different diluents However, neither of these improvements has turned them immune to the hydrolytic degradation that occurs with time. Self-etching adhesive systems produce semi-permeable membranes after polymerization and this permeability allows water leakage, which can be accumulated in the different layers of the adhesive interface, creating porosities that have been recognized as the first signal of interfacial degradation over time $^{4}$, apart from dentin intrinsic humidity, which can also cause hydrolysis.

In the non-treated control group, dentin surface was completely covered by smear layer and all tubules were occluded (presence of smear plugs). A consistent, well defined hybrid layer cannot be formed under these conditions, which justifies dentin conditioning to remove the mineral content of this tissue and prepare the collagen network for adhesive infiltration. A previous scanning electron microscopic study has reported similar results while evaluating the effects of some contemporary adhesive systems on the dentin morphology ${ }^{7}$.
In the present study, the phosphoric acid-etched specimens (group 2) presented a greater depth of demineralization than those conditioned with self-etching adhesive systems (groups 3 and 4) and exhibited surfaces free of smear layer and smear plugs, presenting $100 \%$ of open dentinal tubules. The funnel-shaped aspect of the dentinal tubules was observed solely in this group. In the other groups, the original morphology of the dentinal tubules was preserved. This aspect seems to be related to the $\mathrm{pH}$ of adhesive materials and is characteristic of adhesive systems with lower $\mathrm{pH}$. Although the specimens treated with Clearfil SE Bond (primer acidic) did not present tubules with the funneled aspect, which is typical of acid-etched dentin, they exhibited a peritubular dentin with cuff-like aspect, characterized by the presence of a kind of deeper step in the internal surface. Studies had demonstrated that self-etching adhesive systems with $\mathrm{pH}$ similar to that of phosphoric acid (e.g.: Prompt L Pop) are able to completely solve the smear layer and smear plugs and form a hybrid layer with thickness similar to that formed with etch-and-rinse adhesive systems $^{3,4,9,12}$.

One of the main proposals of the self-etching adhesive systems is to suppress the hybridization deficit that might occur with total-etch adhesive systems represented by an acid-demineralized area that was not properly infiltrated by the adhesive material. However, recent studies have shown that discrepancies between the depth of demineralization and the depth of penetration of resin monomers can occur when self-etching adhesive systems are used. A study analyzed 10 adhesive systems, being 5 all-in-one systems and 5 two-step systems ${ }^{2}$. Areas of conditioned dentin not infiltrated by the adhesive were found below the hybrid layer in both types of adhesive systems. These areas were clearly identified in one-step adhesive systems (Xeno III, iBond, Brush \& Bond) and in the experimental material. In two-step systems (Clearfil SE Bond and Clearfil Protect Bond), non-infiltrated demineralized zones were occasionally found and, when present, they were discontinuous and thin. The authors concluded that the occurrence of conditioned zones, but not hybridized by adhesive monomers, is probably material-dependent and is not exclusive of one-step or twostep systems.

In the present study, Clearfil SE Bond presented a greater depth of demineralization and a higher percentage of open dentinal tubules (around 50\%), revealing more efficiency in removal of smear layer and smear plugs, which is favorable for the formation of an adjusted hybrid layer. On the other hand, in spite of having a similar $\mathrm{pH}$ to that of Clearfil SE Bond, iBond presented a lesser depth of demineralization and was not so efficient in removing the smear layer, leaving some open (approximately 39\%), some partially occluded (approximately 27\%) and some totally occluded (approximately 33\%) dentinal tubules.

It is important to point out that both adhesives present the same $\mathrm{pH}(1,9)$, which suggests that $\mathrm{pH}$ is not the only factor accounting for the alterations produced by conditioners on dentinal substrate. This has also been demonstrated in a previous study ${ }^{5}$, which investigated the 
differences in the hybrid layer formed by self-etching adhesive systems and the influence of primer $\mathrm{pH}$ and concluded that $\mathrm{pH}$ was not the most decisive factor for the obtained differences. There were differences in the interface, mainly referring to hybrid layer thickness, presence or absence of gaps in the adhesive/dentin interface and total or partial sealing of the dentinal tubules.

In view of this, other characteristics of the tested adhesive systems might have contributed to the results reached in the present investigation and should therefore be addressed in future studies.

\section{CONCLUSION}

It may be concluded all tested conditioners were able to change dentin morphology. However, it cannot be stated that the agent aggressiveness was the only cause of the micromorphological alterations because a single morphological pattern was not established for each group, but rather an association of different aspects, according to the aggressiveness of the tested conditioner.

\section{AKNOWLEDGMENT}

The authors would like to thank Prof. Ivo Krejci and his staff (University of Geneve) for technical support in this study.

\section{REFERENCES}

1- Buonocore MG. A simple method of increasing the adhesion of acrylic filling materials to enamel surfaces. J Dent Res. 1955;34:84953.

2- Carvalho RM, Chersoni S, Frankenberger R, Pashley DH, Prati C, Tay FR. A challenge to the conventional wisdom that simultaneous etching and resin infiltration always occurs in self-etch adhesives. Biomaterials. 2005;26:1035-42.

3- Chersoni S, Suppa P, Grandini S, Goracci C, Monticelli F, Yiu C, et al. In vivo and in vitro permeability of one-step self-etch adhesives. J Dent Res. 2004;83(6):459-64.

4- Gregoire G, Millas A. Microscopic evaluation of dentin interface obtained with 10 contemporary self-etching systems: correlation with their pH. Oper Dent. 2005;30(4):481-91.

5- Koibuchi H, Yasuda N, Nakabayashi N. Bonding to dentin with a self-etching primer: the effect of smear layers. Dent Mater. $2001 ; 17: 122-6$

6- Lopes GC, Baratieri LN, Andrade MAC, Vieira LCC. Dental adhesion: present state of the art and future perspective. Quintessence Int. $2002 ; 33(3): 213-24$.

7- Montes MAJR, Goes MF, Sinhoreti MAC. The in vitro morphological effects of some current pre-treatments on dentin surface: a SEM evaluation. Oper Dent. 2005;30(2):201-12.

8- Nakabayashi N, Kojima K, Masuhara E. The promotion of adhesion by the infiltration of monomers into tooth substrates. J Biomed Mater Res. 1982;16:165-73.
9- Oliveira SSA, Pugach MK, Hilton JF, Watanabea LG, Marshall SJ, Marshall Jr GW. The influence of the dentin smear layer on adhesion: a self-etching primer vs. a total-etch system. Dent Mater. 2003;19:758-67.

10- Perdigão J, Ramos JC, Lambrechts P. In vitro interfacial relationship between human dentin and one-bottle dental adhesives. Dent Mater. 1997;13:218-27.

11- Schilke R, Lisson JA, Bauss O, Geurtsen W. Comparison of the number and diameter of dentinal tubules in human and bovine dentine by scanning electron microscopic investigation. Arch Oral Biol. 2000;45:355-61.

12- Tay FR, Pashley DH. Aggressiveness of contemporary selfetching systems. I: depth of penetration beyond dentin smear layers. Dent Mater. 2001;17(4):296-308.

13- Tay FR, Pashley DH. Have dentin adhesives become too hydrophilic? J Can Dent Assoc. 2003;11:726-31.

14- Tay FR, Sano H, Carvalho R, Pashley EL, Pashley DH. An ultrastructural study of the influence of acidity of self-etching primers and smear layer thickness on bonding to intact dentin. J Adhes Dent. 2000;2(2):83-98.

15- Van Meerbeek B, De Munck J, Yoshida Y, Inoue S, Vargas M, Vijay $\mathrm{P}$, et al. Adhesion to enamel and dentin: current status and future challenges. Oper Dent. 2003;28(3):215-35.

16- Wang Y, Spencer P. Continuing etching of an all-in-one adhesive in wet dentin tubules. J Dent Res. 2005;84(4):350-4.

17- Watanabe I, Nakabayashi M, Pashley DH. Bonding to ground dentin by a Phenyl-P self-etching primer. J Dent Res.1994;73:121220 . 